\title{
Bayes meets Tikhonov: Understanding Uncertainty within Gaussian Framework for Seismic Inversion
}

\author{
Muhammad Izzatullah ${ }^{1}$, Daniel Peter ${ }^{1}$, \\ Sergey Kabanikhin ${ }^{2,3}$, Maxim Shishlenin ${ }^{2,3}$ \\ ${ }^{1}$ Seismic Modeling and Inversion Group,
} King Abdullah University of Science and Technology, 23955 Thuwal, Saudi Arabia

2 Institute of Computational Mathematics and Mathematical Geophysics SB RAS, 630090 Novosibirsk, Russia

3 Novosibirsk State University, 630090 Novosibirsk, Russia

November 12, 2020 


\title{
Chapter 8
}

\section{Bayes meets Tikhonov: Understanding Uncertainty within Gaussian Framework for Seismic Inversion}

\author{
Muhammad Izzatullah ${ }^{1}$, Daniel Peter ${ }^{1}$, Sergey Kabanikhin ${ }^{2,3}$, Maxim Shishlenin ${ }^{2,3}$
}

In this chapter, we demonstrate the sound connection between the Bayesian approach and the Tikhonov regularisation within Gaussian framework. We provide an uncertainty analysis framework to answer the following two fundamental questions: (1) How well is the estimate determined by the a posteriori PDF, i.e. by the combination of observed data and a priori information? (2) What are the respective contributions of observed data and a priori information? To support the proposed methodology, we demonstrate it through numerical application in linear inverse problem for the seismic travel-time tomography.

\subsection{Introduction}

The objective of seismic inversion is to provide information about the internal structure of the Earth from observed data. In most geophysical applications, seismic inversion is introduced as an iterative, local optimisation problem that attempts to minimise the least-squares residuals between observed and synthetic data. Mathematically, the inverse problem is ill-posed $[1,2,3,4,5,6,7]$, leading to a non-uniqueness of the solutions. It remains challenging to solve inverse problems practically due to limitations in data acquisition, measurement uncertainties and the non-uniqueness of the solution. Several advancements in geophysics have been proposed to tackle these challenges $[8,9,10,11,12]$. Despite these rapid advancements, the research on uncertainty analysis for the seismic inversion solutions is progressing slowly due to limitations of computational power and algorithms advancement.

Uncertainty analysis conventionally performs within the Bayesian framework. Within the geophysics community, uncertainty analysis commonly refers to as the resolution analysis [13, 14, 15]. Backus and Gilbert [16, 17] developed the concept of resolution for linear geophysical inverse problems. Although this concept is not an original concept for uncertainty analysis within the Bayesian framework, we can extend its definition to 
accommodate the framework. Hence, the resolution defines a balance measurement between the information from the observed data in comparison with the a priori information in determining the parameter estimates $[18,19,20,21,22]$. One should notice that by this definition, parameters can be well resolved by the entire state of information while being poorly resolved from the observed data. In practice, the observed seismic data typically are finite and corrupted by noise. The main consequence of these features is that the observed data may be informative, relative to the a priori information, only on a low-dimensional subspace of the entire parameter space [23, 8, 9, 24, 25].

Focusing back in the Bayesian framework, firstly the parameters of interest are estimated by combining the information from observed data with a priori information on the parameters. The information from observed data reflects in the likelihood function, and the a priori information on the parameters imbeds in the a priori probability density function $(\mathrm{PDF})$. The product of the two determines the a posteriori PDF which is the solution to the inverse problem within the Bayesian framework. The uncertainty analysis can be performed through sampling the a posteriori PDF with the family of Monte Carlo methods [26, 20, 27]. Through the accepted samples, we can infer the parameter fields and conduct the uncertainty analysis. However, for a large-scale inverse problem, e.g. seismic inversion, the family of Monte Carlo methods are infeasible and impractical. As an alternative, the most trivial approach to inspecting the a posteriori PDF through the whole of parameter space, its maximum is taken as a point estimate of the parameters of interest. This approach is known as the Laplace approximation in the statistical literature $[28,29,30]$. It is based on a second-order Taylor approximation of the log posterior around the maximum a posteriori point (MAP) estimate, which results in a Gaussian approximation to the posterior. However, such a point estimate contains a limited amount of information; hence, it only infers uncertainty about a point instead of the whole parameter space.

Despite the fact, the Laplace approximation approach has strong ties with the field of optimisation in estimating the MAP. The problems of estimating the MAP can be simplified into minimising a misfit function of the negative logarithm of a posteriori PDF. The negative logarithm of a posteriori PDF resembles the general Tikhonov regularisation misfit function $[31,32,33,34]$. This simplification enables us to infer the uncertainty at the MAP point.

Based on our extended definition of the concept of resolution, the uncertainty analysis problem is essentially one of information reduction. This information reduction reflects in the standard deviations or variances of the parameters contains in the a posteriori covariance matrix. These can be interpreted as overall uncertainty bounds in the directions along which the parameters are poorly resolved. Although this is the most convenient to analyse the uncertainty, note that this a posteriori covariance matrix is evaluated at the MAP point; thus, the form of information is limited.

In this chapter, we demonstrate the sound connection between the Bayesian approach and the Tikhonov regularisation within Gaussian framework. We provide an uncertainty analysis framework to answer the following two fundamental questions: (1) How well is the estimate determined by the a posteriori PDF, i.e. by the combination of observed data and a priori information? (2) What are the respective contributions of observed 
data and a priori information? To support the proposed methodology, we demonstrate it through numerical application in linear inverse problem for the seismic travel-time tomography.

\subsection{Regularisation method of A. N. Tikhonov}

We start this chapter by introducing the Tikhonov regularisation technique. From the perspective of inverse and ill-posed problems, we can pose the problems as $A m=d$, where $m$ represents the model parameter, and $d$ is the observed data. We define $\mathcal{M}$ and $\mathcal{D}$ as metric spaces of model parameter and data, respectively. $A$ is an operator $A: \mathcal{M} \rightarrow \mathcal{M}$, which maps $m$ from model parameter space $\mathcal{M}$ onto data space $\mathcal{D}$.

In many ill-posed problems, $A m=d$ has a class of possible solutions $\tilde{\mathcal{M}} \subset \mathcal{M}$ which is not compact, but due to the data $d$ contains measurement errors, the output solution $A(\tilde{\mathcal{M}})$ lies beyond the existence class. For constructing the approximate solutions to such problems, A. N. Tikhonov $(1963,1964)$ proposed a regularisation technique. Let $m_{e}$ to be the exact solution of an ill-posed problem $A m=d$ for some $d \in \mathcal{D}$.

Definition (Regularising Family). The family of operators $\left\{\mathcal{R}_{\alpha}\right\}_{\alpha>0}$ is called regularising for the problem $A m=d$ if

1) for any $\alpha>0$ operator $\mathcal{R}_{\alpha}: \mathcal{D} \rightarrow \mathcal{M}$ continuous,

2) for any $\varepsilon>0$, there is $\alpha_{*}>0$ such that for all $\alpha \in\left(0, \alpha_{*}\right)$

$$
\rho_{\mathcal{M}}\left(\mathcal{R}_{\alpha} d, m_{e}\right)<\varepsilon
$$

other word,

$$
\lim _{\alpha \rightarrow+0} \mathcal{R}_{\alpha} d=m_{e}
$$

If the right side of the equation $A m=d$ is given approximately and the error of the original data $\rho_{\mathcal{D}}\left(d_{\delta}, d\right) \leq \delta$ is known, then the regularising family $\left\{\mathcal{R}_{\alpha}\right\}_{\alpha>0}$ allows not only to build an approximate solution $m_{\alpha \delta}=\mathcal{R}_{\alpha} d_{\delta}$, but also evaluate the evasion of the approximate solution $m_{\alpha \delta}$ from the exact $m_{e}$. In fact, by virtue of the triangle inequalities,

$$
\rho_{\mathcal{M}}\left(m_{\alpha \delta}, m_{e}\right) \leq \rho_{\mathcal{M}}\left(m_{\alpha \delta}, \mathcal{R}_{\alpha} d\right)+\rho_{\mathcal{M}}\left(\mathcal{M}_{\alpha} d, m_{e}\right)
$$

When $\alpha \rightarrow+0$, the second term on the right-hand side (8.2.2) tends to zero. Due to the ill-posedness of the problem, to estimate the first term for $\alpha \rightarrow+0, \delta \rightarrow+0$ is a complex problem. However, this can be solved by taking into account the a priori and/or a posteriori information about the exact solution.

Consider the case where $\mathcal{M}, \mathcal{D}$ are Banach spaces as an example. Let $A: \mathcal{M} \rightarrow \mathcal{D}$ as a linear, completely continuous operator, and $\mathcal{R}_{\alpha}$ as a linear operator for all $\alpha>0$. Assume that for $d \in \mathcal{D}$ there is a single solution $m_{e}$ and instead of $d$, given its approximation $d_{\delta} \in \mathcal{D}$ such that 


$$
\left\|d-d_{\delta}\right\| \leq \delta
$$

We estimate the norm of the difference between the exact solution $m_{e}$ and the regularised one $m_{\alpha \delta}=\mathcal{R}_{\alpha} d_{\delta}$ by

$$
\left\|m_{e}-m_{\alpha \delta}\right\| \leq\left\|m_{e}-\mathcal{R}_{\alpha} d\right\|+\left\|\mathcal{R}_{\alpha} d-\mathcal{R}_{\alpha} d_{\delta}\right\| .
$$

Denote $\left\|m_{e}-\mathcal{R}_{\alpha} d\right\|=\gamma\left(m_{e}, \alpha\right)$. By virtue of the property (8.2.1) of the regularising family, the first term on the right-hand side of (8.2.4) tends to zero when $\alpha \rightarrow 0$, i.e., $\lim _{\alpha \rightarrow 0} \gamma\left(m_{e}, \alpha\right)=0$. From the linearity of $\mathcal{R}_{\alpha}$ and the condition (8.2.3) it follows that

$$
\left\|\mathcal{R}_{\alpha} d-\mathcal{R}_{\alpha} d_{\delta}\right\| \leq\left\|\mathcal{R}_{\alpha}\right\| \delta
$$

Recall that the norm of the operator $A: \mathcal{M} \rightarrow \mathcal{D}$, where $\mathcal{M}$ and $\mathcal{D}$ are Banach spaces, defined by the formula

$$
\|A\|=\sup _{\substack{m \in \mathcal{M} \\ m \neq 0}} \frac{\|A m\|}{\|m\|} .
$$

Because the problem is ill-posed, the norm $\left\|\mathcal{R}_{\alpha}\right\|$ cannot be uniformly bounded, otherwise $\lim _{\alpha \rightarrow+0} \mathcal{R}_{\alpha}=A^{-1}$, and the problem $A m=d$ would be classically well-posed. However, if $\alpha$ and $\delta$ tend to zero consistently, then the right-hand side of the received estimate

$$
\left\|m_{e}-m_{\alpha \delta}\right\| \leq \gamma\left(m_{e}, \alpha\right)+\left\|\mathcal{R}_{\alpha}\right\| \delta
$$

approach zero. In fact, let's denote $\omega\left(m_{e}, \delta\right)=\inf _{\alpha>0}\left\{\gamma\left(m_{e}, \alpha\right)+\left\|\mathcal{R}_{\alpha}\right\| \delta\right\}$ and we show that

$$
\lim _{\delta \rightarrow 0} \omega\left(m_{e}, \delta\right)=0
$$

Let $\varepsilon>0$ be an arbitrary number. Because $\lim _{\alpha \rightarrow 0} \gamma\left(m_{e}, \alpha\right)=0$, then there is such an $\alpha_{0}(\varepsilon)$ that for all $\alpha \in\left(0, \alpha_{0}(\varepsilon)\right)$

$$
\gamma\left(m_{e}, \alpha\right)<\varepsilon / 2 \text {. }
$$

Denote $\mu_{0}(\varepsilon)=\inf _{\alpha \in\left(0, \alpha_{0}(\varepsilon)\right)}\left\|\mathcal{R}_{\alpha}\right\|$ and take $\delta_{0}(\varepsilon)=\varepsilon /\left(2 \mu_{0}(\varepsilon)\right)$. Then for $\delta \in\left(0, \delta_{0}(\varepsilon)\right)$

$$
\inf _{\alpha>0}\left\{\left\|\mathcal{R}_{\alpha}\right\| \delta\right\} \leq \delta \inf _{\alpha \in\left(0, \alpha_{0}(\varepsilon)\right)}\left\{\left\|\mathcal{R}_{\alpha}\right\|\right\} \leq \varepsilon / 2 .
$$

Therefore, for an arbitrary $\varepsilon>0$, we can find $\alpha_{0}(\varepsilon)$ and $\delta_{0}(\varepsilon)$ such that for all $\alpha \in\left(0, \alpha_{0}(\varepsilon)\right)$ and $\delta \in\left(0, \delta_{0}(\varepsilon)\right)$

$$
\left\|m_{e}-m_{\alpha \delta}\right\|<\varepsilon .
$$


We have defined the regularisation family, and now let us introduce the Tikhonov functional, a well-known approach in regularisation technique, which can be described as

$$
T\left(m, d_{\delta}, \alpha\right)=\left\|A m-d_{\delta}\right\|+\alpha \Omega(m) .
$$

For specific operators $A$ and regularisation families $\left\{\mathcal{R}_{\alpha}\right\}_{\alpha>0}$, the relationship between the regularisation parameter $\alpha$ and the level errors $\delta$ in the observed data can be obtained explicitly.

To construct a regularisation family, we need to choose a sequence of $\alpha_{n}$ where $\alpha_{n} \rightarrow 0$ as $n$ goes to infinity. This sequence is called the regularisation sequence. Consider an example, let $\mathcal{D}=\mathcal{M}$, where $\mathcal{M}$ is a separable Hilbert space. Let $A$ be a linear operator, a completely continuous self-adjoint positive, and $\left\{\varphi_{n}\right\},\left\{\lambda_{n}\right\}$ are the corresponding sequences of eigenfunctions and eigenvalues of operator $A\left(\lambda_{k+1} \leq \lambda_{k}, k \in \mathbb{N}\right)$. The exact solution $m_{e}$ of the equation $A m=d$ is can be represented in the form

$$
m_{e}=\sum_{k=1}^{\infty} \frac{d_{k}}{\lambda_{k}} \varphi_{k}, \quad d_{k}=\left\langle d, \varphi_{k}\right\rangle .
$$

Since the solution exists and belongs to a Hilbert space $\mathcal{M}$, the series (8.2.6) and $\sum_{k=1}^{\infty}\left(d_{k} / \lambda_{k}\right)^{2}$ converge. But, for $d_{\delta} \in D$, if there is no condition that satisfies $\left\|d-d_{\delta}\right\|<\delta$ for the solutions of the equation $A m=d_{\delta}$, then the series $\sum_{k=1}^{\infty}\left(d_{\delta k} / \lambda_{k}\right)^{2}, d_{\delta k}=\left\langle d_{\delta}, \varphi_{k}\right\rangle$, diverges[34]. Consider a sequence of operators $\left\{\mathcal{R}_{n}\right\}$

$$
\mathcal{R}_{n} d_{\delta}=\sum_{k=1}^{n} \frac{d_{\delta k}}{\lambda_{k}} \varphi_{k}
$$

and we show that it has all the properties of a regularising families at $n \rightarrow \infty$. The $\mathcal{R}_{n}$ operators are obviously continuous, $\left\|\mathcal{R}_{n}\right\|=1 / \lambda_{n}$ and for all $m \in M$

$$
\lim _{n \rightarrow \infty} \mathcal{R}_{n} A m=\lim _{n \rightarrow \infty} \sum_{k=1}^{n} m_{k} \varphi_{k}=m, \quad m_{k}=\left\langle m, \varphi_{k}\right\rangle .
$$

But then for the exact solution (8.2.6) of the equation $A m=d$ and a regularised solution (8.2.7) $m_{\delta n}=\mathcal{R}_{n} d_{\delta}$ can be fairly approximate by

$$
\begin{aligned}
\left\|m_{e}-m_{\delta n}\right\| \leq\left\|m_{e}-\mathcal{R}_{n} d\right\|+\left\|\mathcal{R}_{n} d-\mathcal{R}_{n} d_{\delta}\right\| & \leq\left\|m_{e}-\mathcal{R}_{n} A m_{e}\right\|+\left\|\mathcal{R}_{n}\left(d-d_{\delta}\right)\right\| \\
& \leq \sum_{k=n+1}^{\infty} m_{k}^{2}+\left\|\mathcal{R}_{n}\right\| \delta=\sum_{k=n+1}^{\infty} m_{k}^{2}+\frac{\delta}{\lambda_{n}} .
\end{aligned}
$$

Therefore, for any $\varepsilon>0$, we may first select number $n_{0}$ such that

$$
\sum_{k=n_{0}+1}^{\infty} m_{k}^{2}<\varepsilon / 2
$$

and then select $\delta>0$ from the condition $\delta<\lambda_{n} \varepsilon / 2$. Then, for all $n>n_{0}$ and $\delta \in\left(0, \lambda_{n} \varepsilon / 2\right)$, we obtained a completed inequality $\left\|m_{e}-m_{\delta n}\right\|<\varepsilon$. 
Now, we introduce the sequential approximation method. Let all the conditions of the previous example be met and, additionally, $\lambda_{1}<1$. Let's define the sequence $\left\{m_{n}\right\}$ by the rule

$$
m_{n+1}=m_{n}-A m_{n}+d, m_{0}=d, n=0,1,2, \ldots
$$

We show that if there is an exact solution to the equation $A m=d, m_{e} \in \mathcal{M}$, then

$$
\lim _{n \rightarrow \infty} m_{n}=m_{e}
$$

Indeed,

$$
m_{n}=\sum_{k=0}^{n}(E-A)^{k} d=\sum_{k=0}^{n}(E-A)^{k} A m_{e} .
$$

Consider now the regularising family, which is built on the basis of minimizing the Tikhonov functional

$$
T(m, d, \alpha)=\|A m-d\|^{2}+\alpha \Omega(m),
$$

where $\Omega(m)$ is continuous non-negative convex functional (stabilizing functional Tikhonov, Arsenin, 1974), satisfying certain conditions. Consider the simplest example of the Tikhonov functional

$$
T(m, d, \alpha)=\|A m-d\|^{2}+\alpha\|m\|^{2}, \quad \alpha>0 .
$$

Theorem 1. Let $\mathcal{M}$ and $\mathcal{D}$ be separable Hilbert spaces, $A$ - linear completely continuous operator. Then for all $d \in\lceil$ and $\alpha>0$ functional $T(m, d, \alpha)$ reaches its lower edge on a single element $m_{\alpha}$.

The proof is presented, for example, in the following book (Denisov, 1994). We will discuss only some of its stages. First, the regularisation term enforces the nonnegativity of the functional in $M$. There is an element $m_{0}$, where its minimum $T_{0}=$ $\inf _{m \in M} T(m, d, \alpha)=T\left(m_{0}, d, \alpha\right) \geq 0$ is reached. It is clear that if $T_{0}=0$, then $m_{0}$ will be the solution of the equation $A m=d$. To prove the uniqueness of $m_{0}$, we may write the increment of the functional as

$$
\begin{aligned}
T(m+\delta m, d, \alpha)-T(m, d, \alpha) & =2\langle A m-d, A \delta m\rangle+2 \alpha\langle m, \delta d\rangle+\langle A \delta m, A \delta m\rangle+\alpha\langle\delta m, \delta m\rangle \\
= & 2\left\langle A^{*} A m-A^{*} d+\alpha m, \delta m\right\rangle+\langle A \delta m, A \delta m\rangle+\alpha\langle\delta m, \delta m\rangle .
\end{aligned}
$$

Here $A^{*}$ is the conjugate operator to $A$.

From the last inequality, by taking into account the approximation of

$$
\mid\langle A \delta m, A \delta m\rangle+\alpha\langle\delta m, \delta m\rangle \leq\left(\left\|A^{2}\right\|+\alpha\right)\|\delta m\|^{2},
$$

it follows that for any $m \in \mathcal{M}$, the functional $T(m, d, \alpha)$ has a gradient of the form

$$
T^{\prime}(m, d, \alpha)=2\left(A^{*} A m-A^{*} d+\alpha m\right) .
$$

Therefore, $m_{0}$ is the solution of the equation 


$$
\alpha m+A^{*} A m=A^{*} d .
$$

By virtue of the linearity of the operator $A$, it is sufficient to prove that homogeneous equation

$$
\alpha m+A^{*} A m=0
$$

has only the trivial solution to guarantee the uniqueness of solution in the equation (8.2.9). We can show this through contradiction. That is, assume that there is a non-zero element $\bar{m} \in \mathcal{M}$ such that $\alpha \bar{m}+A^{*} A \bar{m}=0$. But, then the equality $\left\langle\alpha \bar{m}+A^{*} A \bar{m}, \alpha \bar{m}+A^{*} A \bar{m}\right\rangle=0$ can be rewritten in the form

$$
\alpha^{2}\langle\bar{m}, \bar{m}\rangle+2 \alpha\langle A \bar{m}, A \bar{m}\rangle+\left\langle A^{*} A \bar{m}, A^{*} A \bar{m}\right\rangle=0 .
$$

This is a contradiction, because in the left part of the latter inequalities the first term is strictly positive, and the other two are non-negative. Thus, we show the uniqueness of solution in the equation (8.2.9).

For an approximated observed data $d_{\delta} \in \mathcal{D}$ which satisfies the condition $\left\|d-d_{\delta}\right\| \leq \delta$, there is an approximate solution $m_{\alpha \delta}$ where its converge to the exact solution $m_{e}$ of the equation $A m=d$ as $\alpha$ and $\delta$ parameters go to zero, respectively. In fact, there exists and unique minimum of the functional $T\left(m, d_{\delta}, \alpha\right)$ at the point $m_{\alpha \delta}$, which described by the theorem 1 below.

Theorem 2 (Denisov, 1994). Let the conditions of the Theorem 1 satisfied. Suppose that for some $d \in \mathcal{D}$, there is a single solution $m_{e}$ the equation $A m=d$. Denote $\left\{d_{\delta}\right\}_{\delta>0}$ as a family of approximated data, each element of which satisfies the condition $\left\|d-d_{\delta}\right\|<\delta$. Then, if $\delta$ is chosen tends to zero, the regularisation parameter $\alpha=\alpha(\delta)$ is selected so that $\lim _{\delta \rightarrow 0} \alpha(\delta)=0$ and $\lim _{\delta \rightarrow 0} \delta^{2} / \alpha(\delta)=0$. Then, the element $m_{\alpha(\delta), \delta}$, at which the minimum regularising value is reached by the functional $T\left(m, d_{\delta}, \alpha\right)$, tends to the exact solution $m_{e}$ of the equation $A m=d$, i.e. $\lim _{\delta \rightarrow 0}\left\|m_{\alpha(\delta), \delta}-m_{e}\right\|=0$.

\subsection{Bayesian-Tikhonov Formulation for Linear Inverse Problems}

In this section, we introduce the problem formulation for linear inverse problems in the Bayesian framework. Next, we demonstrate the sound connection between the Bayesian approach and the Tikhonov regularisation, as explained in the previous section. Here, we introduce the derivation of our problem formulation, and interested readers may refer to $[28,29,30,27]$ for more details problem formulation in the Bayesian framework.

Consider a linear system of equations with additive Gaussian noise in the observed data,

$$
\begin{aligned}
& d=A m+e \\
& m \in \mathcal{R}^{n}, \quad d, e \in \mathcal{R}^{m}, \quad A \in \mathcal{R}^{m \times n},
\end{aligned}
$$


and let the parameters $m$ and the observed data $d$ be the random variables. We assume that $m$ and $e$ are independent and identical Gaussian distribution with zero means,

$$
m \sim \mathcal{N}\left(0, \gamma^{2} C_{m}\right), \quad e \sim \mathcal{N}\left(0, \sigma^{2} I\right) .
$$

The covariance of the noise, $\sigma^{2} I$ indicates that each component of $\mathrm{D}$ is contaminated by independent and identically distributed random noise. Now, we define the a priori density of the form

$$
\pi_{\text {prior }}(m)=\exp \left(-\frac{1}{2 \gamma^{2}} m^{T} C_{m}^{-1} m\right),
$$

and the likelihood density by assuming that the noise covariance is known is

$$
\pi_{l i k e}(d \mid m)=\exp \left(-\frac{1}{2 \sigma^{2}}\|d-A m\|^{2}\right) .
$$

By Bayes theorem, we can define our a posterior density by the product of the likelihood and the a priori densities. The a posteriori density defines as

$$
\begin{aligned}
\pi_{\text {post }}(m \mid d) & \propto \pi_{\text {like }}(d \mid m) \pi_{\text {prior }}(m) \\
& \propto \exp \left(-\frac{1}{2 \sigma^{2}}\|d-A m\|^{2}-\frac{1}{2 \gamma^{2}} m^{T} C_{m}^{-1} m\right) \\
& =\exp (-V(m \mid d)),
\end{aligned}
$$

where

$$
V(m \mid d)=\frac{1}{2 \sigma^{2}}\|d-A m\|^{2}+\frac{1}{2 \gamma^{2}} m^{T} C_{m}^{-1} m .
$$

Based on the Gaussian likelihood and a priori densities, the a posteriori density $\pi_{\text {post }}(m \mid d)$ is also Gaussian. This condition is only valid for the linear case, and for the non-linear case, this might not hold.

Next, we demonstrate the sound connection between the Bayesian approach and the Tikhonov regularisation. Consider the matrix $C_{m}$ is symmetric positive definite, so is its inverse; thus, it admits Cholesky factorisation of the form

$$
C_{m}^{-1}=L^{T} L .
$$

With this notation, we can reformulate our $V(m \mid d)$ into the Tikhonov regularisation functional, defines as

$$
T(m)=2 \sigma^{2} V(m \mid d)=\|d-A m\|^{2}+\lambda^{2}\|L m\|^{2}, \quad \lambda=\frac{\sigma}{\gamma},
$$

where $\lambda$ is the ratio of the noise and the a priori variances. It also acts as a parameter which balancing the information contributions between the likelihood and the a priori densities.

The Tikhonov regularisation functional describes in equation (8.3.8), plays a crucial role in the classical regularisation theory. From the Tikhonov regularisation perspectives, 
the first term controls the closeness of the solution to the observed data and the second one acts as a penalty. The regularisation matrix $L$ is typically selected so that large $\|L m\|$ corresponds to an undesirable feature of the solution $[31,33,32,34]$. In order to provide a balance between these two terms, the choice of the $\lambda$ is a non-trivial challenge in Tikhonov regularisation. In general, Tikhonov regularisation solution is the outcome of a balance between fitting the data and eliminating unwanted features. As we have observed, the Tikhonov regularisation fits well into the Bayesian framework, since its interpretation parallels to the concept of likelihood and a priori densities.

\subsection{The Maximum A Posteriori (MAP) Point}

In the previous section, we have demonstrated the sound connection between the Bayesian approach and the Tikhonov regularisation. In this section, we discuss the MAP point estimation of the a posteriori density.

The MAP estimate is the point in the parameter space that maximises the a posteriori probability density function $(\mathrm{PDF})$. The problems of estimating the MAP can be simplified into minimising a misfit function of the negative logarithm of the a posteriori PDF. The negative logarithm of a posteriori PDF resembles the general Tikhonov regularisation misfit function, as shown in equation (8.3.8) in the previous section. The Maximum A Posteriori (MAP) estimator defines as,

$$
\begin{aligned}
m_{M A P} & =\arg \max _{m} \pi_{\text {post }}(m \mid d) \\
& =\arg \min _{m}-\log \pi_{\text {post }}(m \mid d) \\
& =\arg \min _{m} V(m \mid d) .
\end{aligned}
$$

In this particular case, the MAP estimator is better describes as

$$
m_{M A P}=\arg \min _{m}\left(\|d-A m\|^{2}+\lambda^{2}\|L m\|^{2}\right) .
$$

\subsection{A Generalised Formulation}

To prepare ourselves for the uncertainty analysis, introducing a generalised problem formulation is essential. This generalised problem formulation should fit a balance measurement between the information from the observed data in comparison with the a priori information in determining the parameter estimates. The objective here is to express the parameters in the dimensionless unit so that it is scaling invariant under a linear transformation as proposed by Albert Tarantola [18, 19, 20]. By doing so, our uncertainty analysis is consistent with the units.

In this section, we generalise the formulation of the negative logarithm of a posteriori $\mathrm{PDF}$ in equation (8.3.8). We let the parameter $\lambda$ equals unity, and statistically normalise the parameters with the linear transformation so that the transformed parameters have the identity matrix as a priori covariance matrix. This approach is similar in spirit to the 
standard Tikhonov regularisation functional [31, 32, 33, 34], and the concept of priorpreconditioned data misfit Hessian as proposed in $[23,8,9,10]$.

Consider the following formulation of the negative logarithm of a posteriori PDF

$$
\begin{aligned}
T(m) & =-\log \pi_{\text {post }}(m \mid d) \\
& =\frac{1}{2}\|d-A m\|_{C_{D}}^{2}+\frac{1}{2}\|m-\bar{m}\|_{C_{\text {prior }}}^{2},
\end{aligned}
$$

where $\|x\|_{C}=x^{T} C^{-1} x$. The data noise and a priori covariance matrices are presented by $C_{D}$ and $C_{\text {prior }}$, respectively. At $\nabla T(m)=0$, we can determine the MAP estimator through the following derivations:

$$
\begin{aligned}
\nabla T(m) & =A^{T} C_{D}^{-1}(A m-d)+C_{\text {prior }}^{-1}(m-\bar{m}) \\
& =0
\end{aligned}
$$

Consider that $C_{D}$ and $C_{\text {prior }}$ are invertible symmetric positive definite matrices, and they admit the Cholesky factorisation in the form of

$$
C_{D}=C_{D}^{1 / 2} C_{D}^{1 / 2}, \quad C_{\text {prior }}=C_{\text {prior }}^{1 / 2} C_{\text {prior }}^{1 / 2}
$$

Now we proceed with

$$
\begin{aligned}
\left(A^{T} C_{D}^{-1} A+C_{\text {prior }}^{-1}\right) m & =A^{T} C_{D}^{-1} d+C_{\text {prior }}^{-1} \bar{m} \\
\left(C_{\text {prior }}^{1 / 2} A^{T} C_{D}^{-1 / 2} C_{D}^{-1 / 2} A C_{\text {prior }}^{1 / 2}+I\right) C_{\text {prior }}^{-1 / 2} m & =C_{\text {prior }}^{1 / 2} A^{T} C_{D}^{-1} d+C_{\text {prior }}^{1 / 2} C_{\text {prior }}^{-1} \bar{m} \\
\left(\left(C_{D}^{-1 / 2} A C_{\text {prior }}^{1 / 2}\right)^{T}\left(C_{D}^{-1 / 2} A C_{\text {prior }}^{1 / 2}\right)+I\right) C_{\text {prior }}^{-1 / 2} m & =\left(C_{\text {prior }}^{1 / 2} A^{T} C_{D}^{-1 / 2}\right) C_{D}^{-1 / 2} d+C_{\text {prior }}^{-1 / 2} \bar{m} \\
\left(\tilde{A}^{T} \tilde{A}+I\right) \tilde{m} & =\tilde{d}+\tilde{\bar{m}},
\end{aligned}
$$

where $\tilde{A}=C_{D}^{-1 / 2} A C_{\text {prior }}^{1 / 2}$ and $\tilde{m}=C_{\text {prior }}^{-1 / 2} m$. We define $\tilde{d}=\tilde{A}^{T} C_{D}^{-1 / 2} d$ as the weighted noise-normalized data, and $\tilde{\bar{m}}=C_{\text {prior }}^{-1 / 2} \bar{m}$ as the weighted a priori mean.

As we have observed in equation (8.5.4), our problem formulation has reduced into the standard Tikhonov regularisation functional through prior-preconditioned data misfit Hessian approach. In this formulation, we incorporate an a priori mean $\bar{m}$ and thus introduces bias into the MAP estimate towards this $\bar{m}$. Although such an a priori mean $\bar{m}$ can have a dramatic effect on the MAP estimate, for simplicity of formulation onwards, we consider it equals zero.

We consider the SVD approach as a means to estimate the MAP point. We will introduce the concept of filter factors [31, 33] as a balance measurement between the information from the observed data in comparison with the a priori information in determining the parameter estimates. Through this approach, we can understand these aspects in more detail and can assist us in our uncertainty analysis. 
Consider implementing SVD on matrix $\tilde{A}=U \Sigma V^{T}$, we can rewrite the formulation in equation (8.5.4) and define our MAP estimator as follows:

$$
\begin{aligned}
\left(\tilde{A}^{T} \tilde{A}+I\right) \tilde{m} & =\tilde{d} \\
\left(V \Sigma U^{T} U \Sigma V^{T}+I\right) \tilde{m} & =\tilde{d} \\
\left(V \Sigma^{2} V^{T}+I\right) \tilde{m} & =\tilde{d} \\
V\left(\Sigma^{2}+I\right) V^{T} \tilde{m} & =\tilde{d},
\end{aligned}
$$

and we can define our MAP estimator for this generalised formulation as

$$
\begin{aligned}
\tilde{m}_{M A P} & =V\left(\Sigma^{2}+I\right)^{-1} V^{T} \tilde{d} \\
& =\sum_{i=1}^{n} f_{i} \frac{v_{i}^{T} \tilde{d}}{\sigma_{i}^{2}} v_{i} .
\end{aligned}
$$

The singular values of $\tilde{A}^{T} \tilde{A}$ denotes by $\sigma_{i}^{2}$. The function $f_{i}=\frac{\sigma_{i}^{2}}{\sigma_{i}^{2}+1}$ is the filter factor which acts as the balance measurement between the information from the observed data in comparison with the a priori information in determining the parameter estimates. The filter factor satisfy the following conditions:

$$
f_{i}=\frac{\sigma_{i}^{2}}{\sigma_{i}^{2}+1} \approx \begin{cases}1, & \sigma_{i}^{2} \gg 1, \\ 0, & \sigma_{i}^{2} \ll 1 .\end{cases}
$$

We observe that for singular values $\sigma_{i}^{2}$ larger than 1 , the filter factors are close to one. The corresponding SVD components define the well-resolved or certainty directions concerning the observed data in comparison with the a priori information. On the other hand, for singular values much smaller than 1 , the filter factors are small. The corresponding SVD components define the poor-resolved or uncertainty directions. In these directions, a priori information over-influence the a posteriori PDF than the likelihood PDF does $[22,32,34,25]$. We note that the optimal balance measurement between the information from the observed data in comparison with the a priori information is at $\sigma_{k}^{2}=1$, where $k$ is the index of optimal truncation of singular values. By quoting Albert Tarantola $[18,19,20]$, the high certainty directions in the model space are that have a maximum ratio between the a posteriori information and the a priori. We will return to this concept of filter factor in later sections since it is essential to the analysis a posteriori covariance and resolution matrices.

\subsection{The A Posteriori Covariance Matrix}

In the previous section, we introduced the standard and the generalised problem formulation in the Bayes-Tikhonov framework. In the next two sections, we discuss the a posteriori covariance and resolution matrices for analysis of uncertainties.

In this work, we focus on the framework within Gaussian assumptions based on the Laplace approximation, which apply to both linear and non-linear cases. The Laplace approximation takes on a second-order Taylor approximation of the log posterior around the 
MAP estimate, which results in a Gaussian approximation to the posterior [28, 29, 30]. Although this is the most convenient to analyse the uncertainty, note that this a posteriori covariance matrix is evaluated at the MAP point; thus, the form of information is limited.

For the non-linear case, i.e. non-linear forward model, an exact computation of the a posteriori covariance matrix would involve a full computation of the a posteriori PDF through the whole parameter space. However, this is too computationally demanding in practice even for the most trivial cases. By using Laplace approximation, we can linearise our non-linear forward model about the MAP point resulting the a posteriori PDF looks like a Gaussian distribution with mean $m_{M A P}$ and covariance matrix $C_{\text {post }}=H\left(m_{M A P}\right)^{-1}$, where $H\left(m_{M A P}\right)$ denotes the Hessian at MAP point $[22,20,9,10]$.

Follwing $[23,8,9,10]$, assuming that the forward model $A(m)$ is differentiable, we linearise the right-hand side of equation (8.3.1) around $m_{M A P}$ to obtain

$$
d \approx A\left(m_{M A P}\right)+\frac{\partial A(m)}{\partial m}\left(m-m_{M A P}\right)+e
$$

where $\frac{\partial A(m)}{\partial m}$ is the derivative of $A(m)$ evaluated at $m_{M A P}$. Consequently, this gives the approximation of the negative logarithm of a posteriori PDF in equation (8.5.1) around the $m_{M A P}$

$$
T(m) \approx T\left(m_{M A P}\right)+\frac{1}{2}\left(m-m_{M A P}\right)^{T} C_{\text {post }}^{-1}\left(m-m_{M A P}\right)
$$

thus we have

$$
\pi_{\text {post }}(m \mid d) \approx \pi_{\text {post }}\left(m_{M A P} \mid d\right) \exp \left(\frac{1}{2}\left(m-m_{M A P}\right)^{T} C_{\text {post }}^{-1}\left(m-m_{M A P}\right)\right) .
$$

Note that this linearisation is a reasonable approximation when the non-linear forward model is nearly linear or mildly non-linear, i.e., the limits of data noise are small and with an abundance of observed data. For the linear case, the Gaussian assumptions describe in equations (8.6.2) and (8.6.3) are exact.

As we discussed in Section 4, we should consider a balance measurement between the information from the observed data in comparison with the a priori information in determining the parameter estimates. We statistically normalise the parameters through the linear transformation so that the transformed parameters have the identity matrix as a priori covariance matrix. Consequently, we express the parameters in the dimensionless unit so that it is scaling invariant under a linear transformation - this approach address in details in $[21,22,20,23,9,10]$ and theoretically supported by [32, 25].

Returning to our generalised formulation, our MAP point for the general case (after transformation) define as

$$
\begin{aligned}
m_{M A P} & =C_{\text {prior }}^{1 / 2} \tilde{m}_{M A P} \\
& =C_{\text {prior }}^{1 / 2} V\left(\Sigma^{2}+I\right)^{-1} V^{T} \tilde{d} \\
& =C_{\text {prior }}^{1 / 2} V\left(\Sigma^{2}+I\right)^{-1} V^{T} C_{\text {prior }}^{1 / 2} A^{T} C_{D}^{-1} d \\
& =C_{\text {post }} A^{T} C_{D}^{-1} d,
\end{aligned}
$$


and the a posteriori covariance is then given by

$$
\begin{aligned}
C_{\text {post }} & =\left(A^{T} C_{D}^{-1} A+C_{\text {prior }}^{-1}\right)^{-1} \\
& =C_{\text {prior }}^{1 / 2}\left(C_{\text {prior }}^{1 / 2} A^{T} C_{D}^{-1 / 2} C_{D}^{-1 / 2} A C_{\text {prior }}^{1 / 2}+I\right)^{-1} C_{\text {prior }}^{1 / 2} \\
& =C_{\text {prior }}^{1 / 2}\left(\tilde{A}^{T} \tilde{A}+I\right)^{-1} C_{\text {prior }}^{1 / 2} \\
& =C_{\text {prior }}^{1 / 2}\left(V \Sigma^{2} V^{T}+I\right)^{-1} C_{\text {prior }}^{1 / 2} \\
& =C_{\text {prior }}^{1 / 2} V\left(\Sigma^{2}+I\right)^{-1} V^{T} C_{\text {prior }}^{1 / 2} \\
& =\tilde{V}\left(\Sigma^{2}+I\right)^{-1} \tilde{V}^{T} \\
& =\sum_{i=1}^{n} \frac{1}{\sigma_{i}^{2}+1} \tilde{v}_{i} \tilde{v}_{i}^{T},
\end{aligned}
$$

where $\tilde{V}=C_{\text {prior }}^{1 / 2} V$. To further understand the balance between the information from the observed data in comparison with the a priori information in a posteriori covariance matrix, we consider two extreme situations as described in $[18,19,22,32]$ as follows:

1. The observed data entirely determines the solutions, i.e. the likelihood function entirely determines the shape of the a posteriori PDF.

In this case, have our a posteriori PDF in the form of

$$
C_{\text {post }}=\left(A^{T} C_{D}^{-1} A\right)^{-1} .
$$

Let's perform SVD analysis on the equation (8.6.6). Consider $C_{D}=\gamma^{2} I$ and $A=U \Sigma V^{T}$,

$$
\begin{aligned}
C_{\text {post }} & =\left(A^{T} C_{D}^{-1} A\right)^{-1} \\
& =\left(\frac{1}{\gamma^{2}}\left(V \Sigma U^{T}\right)\left(U \Sigma V^{T}\right)\right)^{-1} \\
& =\left(\frac{1}{\gamma^{2}} V \Sigma^{2} V^{T}\right)^{-1} \\
& =\sum_{i=1}^{n} \frac{\gamma^{2}}{\sigma_{i}^{2}} v_{i} v_{i}^{T} .
\end{aligned}
$$

In equation (8.6.7) above, the singular vectors direction are pointing towards where the observed data have an enormous influence in an absolute sense, i.e., not relative to or in the absence of the a priori information.

The singular values $\sigma_{i}^{2}$ which comes from the forward modelling are inversely proportional to the noise level $\gamma^{2}$ in the observed data. For the observed data with a significantly low noise level, the parameters are excellently well-resolved with low variance in the a posteriori covariance matrix. However, this is not the case in practice, since the observed data is commonly noisy with a significant level of noise. On the other hand, if the noise level is enormously high, we are unable to resolve the parameters well enough, even with perfect forward modelling. We can evaluate this based on the ratio of noise level to the singular values $\frac{\gamma^{2}}{\sigma_{i}^{2}}$. The a posteriori covariance matrix will remain high in values which reflects high uncertainty. 
2. The a priori information entirely determines the solutions, i.e. the shape of the a priori PDF reflects the shape of a posteriori PDF.

In this case, it reflects that the uncertainty after inversion is as significant as before inversion, i.e., there is almost no significant reduction in the variance, and there is no significant information gain.

Consider equation (8.6.5) as a basis for the analysis in this case, and we restate the equation below,

$$
\begin{aligned}
C_{\text {post }} & =\tilde{V}\left(\Sigma^{2}+I\right)^{-1} \tilde{V}^{T} \\
& =\sum_{i=1}^{n} \frac{1}{\sigma_{i}^{2}+1} \tilde{v}_{i} \tilde{v}_{i}^{T},
\end{aligned}
$$

The singular values $\sigma_{i}^{2}$ in equation (8.6.8) are inversely proportional to the noise level in the observed data (Kindly refer to the Section 4). If we consider that in the extreme of the noise level going to zero, the a posteriori covariance matrix will remain when there are singular values equals zero. There will always remain a term

$$
C_{\text {post }}=\sum_{i \in \mathcal{O}} \tilde{v}_{i} \tilde{v}_{i}^{T}
$$

where $\mathcal{O}$ denotes the set of singular vectors correspond to $\sigma_{i}^{2}=0$. The singular vectors $\tilde{v}_{i}$ along these directions are purely pointing towards a priori information, i.e., the observed data does not provide any meaningful information, no matter how low the noise level is. On the other hand, in the absence of a priori information, when the term 1 in the denominator is absent, the a posteriori covariance matrix becomes very large when some of the singular values are very small as addressed in the previous case.

Based on the two extreme situations described above, the a posteriori covariance matrix should take into account the interactions between the information from the observed data and the a priori information. Accounting for such interaction is a crucial feature of the optimal covariance approximation which will discuss in a later section.

\subsection{The Resolution Matrix}

In this section, we discuss the concept of the resolution matrix. As we mentioned earlier, this concept is not an original concept for uncertainty analysis within the Bayesian framework; however, we can extend its definition to accommodate the framework. We define the resolution as a balance measurement between the information from the observed data in comparison with the a priori information in determining the parameter estimates [18, 19, 20, 21, 22].

Conventionally, the resolution matrix $R$ is defined through the linear relation between the estimated and exact model [16, 17]. Here, we will formulate the resolution matrix in the Bayesian framework by incorporating the a priori information. Assume $d_{\text {exact }}$ as 
exact data in the absence of uncertainty from an artificial exact model parameters $m_{\text {exact }}$, and we define the relationship between exact data and exact model parameters as,

$$
d_{\text {exact }}=A m_{\text {exact }} .
$$

The resolution matrix $R$ is defined through the following linear relation,

$$
m_{M A P}-\bar{m}=R\left(m_{\text {exact }}-\bar{m}\right),
$$

where $\bar{m}=m_{\text {prior }}$ and $R=\left(A^{T} C_{D}^{-1} A+C_{\text {prior }}^{-1}\right)^{-1} A^{T} C_{D}^{-1} A$. We need to stress that this concept of the resolution matrix is only valid within the Laplace approximation, i.e., when the exact model parameters $m_{\text {exact }}$ are close enough to the maximum a posteriori point $m_{M A P}$. In practice, neither we know the exact model parameters or the actual resolution matrix that maps the exact model parameters on the estimated ones. However, we can justify this through a posteriori PDF where the exact model parameters are close to the maximum. Hence, if estimated model parameters have a higher probability of being close to the exact one, it should replace the maximum as the point estimate.

The relationship between the resolution matrix and the a posteriori covariance matrix can be derived within Gaussian framework by manipulating equation (8.6.5) [22, 20, 13], and we get

$$
R=I-C_{\text {post }} .
$$

The relationship stated above is for the statistically normalised model parameters on the a priori information, and we have addressed this in Section 4. The essential features of the resolution matrix are the deviations from the identity matrix. Based on the relationship above, it clearly stated that it is the deviations by the a posteriori covariance matrix.

To further understand this concept and to relate with a posteriori covariance matrix, consider the following extreme situations:

1. For solutions determined entirely by observed data, we have the a posteriori covariance matrix significantly smaller than the a priori covariance matrix, $C_{\text {post }}<C_{\text {prior }}$, hence $R=I$, i.e., we have a full resolution from the observed data.

2. For solutions determined entirely by a priori information, we have the a posteriori covariance matrix equals (or without a significant variance reduction) to the a priori covariance matrix, $C_{\text {post }}=C_{\text {prior }}$ hence $R=0$, i.e., there is no resolution from the observed data at all.

Based on these extreme situations, the resolution matrix is a measure of the balance between the information from the observed data in comparison with the a priori information in determining the parameter estimates, as we defined earlier. In other words, the resolution matrix informs the deviations of the ratio of a posteriori to a priori variance from unity.

The relationship in equation (8.7.3) can contribute more insights through SVD analysis. By combining equation (8.6.5) with equation (8.7.3), we get 


$$
R=I-\sum_{i=1}^{n} \frac{1}{\sigma_{i}^{2}+1} \tilde{v}_{i} \tilde{v}_{i}^{T},
$$

where the low singular values $\sigma_{i}^{2}$ determine the deviations of the resolution matrix $R$ from the ideal shape $I$. Following [20], the interpretation of the traces of matrices $I, R$, and $I-R$ are as follows:

- $\operatorname{trace}(I)=n$, the total number of model parameters,

- $\operatorname{trace}(R)=\sum_{i=1} \frac{\sigma_{i}^{2}}{\sigma_{i}^{2}+1}, \quad$ the number of model parameters determined by the observed data,

- $\operatorname{trace}(I-R)=\sum_{i=1} \frac{1}{\sigma_{i}^{2}+1}, \quad$ the number of model parameters determined by the a priori information.

As we observe, the singular values of $R$ is exactly the filter factor stated in the Section 4, equation (8.5.7). The filter factor is a tool to assist us in understanding which singular components in the information from the observed data in comparison with the a priori information contributing in determining the parameter estimates. Consequently, it provides the balance measure we need in approximating the optimal a posteriori covariance matrix which will be discussed in the next section.

\subsection{The Optimal Low-Rank Approximation of A Pos- teriori Covariance Matrix}

In the previous sections, we introduce the concepts of a posteriori covariance and resolution matrices. We also describe the relationship between these matrices and the filter factor as in the Tikhonov regularisation. The a posteriori covariance matrix only informative in specific directions due to the influence of noise in the observed data and the a priori information, however. In this section, we propose the optimal low-rank approximation of a posteriori covariance matrix. We describe the optimality by the a posteriori covariance matrix should take into account the interactions between the information from the observed data and the a priori information to determine the boundary between information and noise. We propose a threshold criterion based on the filter factor in approximating the optimal low-rank a posteriori covariance matrix.

To obtain the optimal low-rank approximation of a posteriori covariance matrix, firstly we need to identify the boundary between information provided by measurements in the observed data with respect to the prior information and extract the information effectively. For this purpose, SVD analysis is the best approach.

Consider implementing SVD on matrix $\tilde{A}=U \Sigma V^{T}$ as in equation (8.5.5) and we restate the equation below: 


$$
\begin{aligned}
\left(\tilde{A}^{T} \tilde{A}+I\right) \tilde{m} & =\tilde{d} \\
\left(V \Sigma U^{T} U \Sigma V^{T}+I\right) \tilde{m} & =\tilde{d} \\
\left(V \Sigma^{2} V^{T}+I\right) \tilde{m} & =\tilde{d} \\
V\left(\Sigma^{2}+I\right) V^{T} \tilde{m} & =\tilde{d} .
\end{aligned}
$$

In the equation above, $V\left(\Sigma^{2}+I\right) V^{T}$ is a linear mapping from the model space $\tilde{m}$ to data space $\tilde{d}$. The column of $V$ in $\tilde{A}$ spans the model space $\tilde{m}$, and the column of $U$ spans the data space $\tilde{d}$. If the singular values are zero or significantly small, the column of $V$ constructs the null-space, or the model space which models cannot be determined by the observed data. However, if the singular values are significantly large, the corresponding column of $U$ constructs the information space where the observed data spanning this space provide valuable information for the model. The singular values provide us a hint about the boundary between information provided by measurements in the observed data with respect to the prior information. Ideally, there is a clear distinction in the singular values spectrum to identify this boundary. Unfortunately, there is a smooth spectrum of singular values due to noise corruption and computational round-off error. This fact indicates the complexity of the determination of the boundary between information provided by measurements in the observed data with respect to the prior information.

To facilitate the determination of the boundary between information provided by measurements in the observed data with respect to the prior information from the singular values spectrum, we can relate this to the concept of filter factor which introduced in Section 4. Consider a filter factor as in equation (8.5.7), yet now we generalise its conditions into,

$$
f_{i}(\lambda)=\frac{\sigma_{i}^{2}}{\sigma_{i}^{2}+\lambda} \approx \begin{cases}1, & \sigma_{i}^{2} \gg \lambda, \\ 0, & \sigma_{i}^{2} \ll \lambda,\end{cases}
$$

where $\lambda$ resembles the ratio of the noise and the a priori variances. In Tikhonov regularisation, $\lambda$ is the regularisation parameter which needs to be estimated $[31,32,33,34]$.

The filter factor above measures how well-resolved or certain the solution concerning the observed data in comparison with the a priori information, which depends on the value of $\lambda$. Thus, the role of $\lambda$ is essential for the optimality of low-rank approximation as it acts as the threshold in truncating the spectrum of singular values of the inverse of a posteriori covariance matrix. If the truncation of the singular spectrum occurs above the threshold value $\lambda$, some of the information contains within the observed data might not be included, we lose the valuable information. However, if the truncation occurs below the threshold value $\lambda$, the influence of noise and a priori information is visible in the solution. Thus, it leads to the reduction in the resolution and increases the variance in the solution. There are many approaches to estimate the value of lambda; however, it is beyond the scope of this chapter. The readers may kindly refer to [31, 32, 33, 34] for more details upon regularisation parameters estimation. We demonstrate the optimality concept further in the numerical examples. 


\subsection{Numerical Examples}

To further understand the concepts introduced in the previous sections, we demonstrate them in the following numerical examples. In this section, we consider the linear seismic travel-time tomography problem as in [35]. The numerical examples simulate geophysical problems where one records the travel time of seismic waves between sub-surface sources and receivers located either at or below the surface. Our first task is to determine the maximum a posteriori model (MAP) of sub-surface attenuation, or slowness, in the domain represented as $m$, the model parameters by minimising the negative log posterior as in equation (8.5.4). In accomplishing this task, we minimise the negative log posterior with conjugate gradient algorithm [35].

We consider a square domain of size $[0,100] \times[0,100]$ with 75 equally spaced sources located at the right side of the domain, and 100 equally spaced receivers located on the top of the surface and the left side of the domain. The true slowness model is illustrated in Figure 1(a). The dominating frequency of the propagating wave is set to $10 \mathrm{~Hz}$. We consider Gaussian prior with covariance matrix $\mathbf{C}_{\text {prior }}=\left(\lambda \mathbf{L}^{T} \mathbf{L}\right)^{-1}$, where $\lambda$ equals unity and $\mathbf{L}$ is the Laplacian matrix. Then, we approximate the MAP by minimising the negative log posterior with 100 iterations of the conjugate gradient algorithm starting from initial slowness model illustrated in Figure 1(b). The results are illustrated in the Figure 2. In Figure 2, we show the approximation of the MAP of the slowness model, together with the model error norms and residual norms plots. In Figure 2(b), by knowing the true slowness model, we can compute the relative model error norms at each iteration through

$$
r_{\text {model }}^{(k)}=\frac{\left\|m_{e}-m^{(k)}\right\|_{2}}{\left\|m_{e}\right\|_{2}} .
$$

As we can observe, the model error norms start to stabilise at the 70-th iteration, which indicates we reached the optimal MAP model, and the iteration can be terminated. However, in real-life applications of inverse problems, the true model is unknown, and the computation of model error norms are impossible. Thus, we need to rely upon other relative norms to guarantee our convergence. Typically, the relative residual norms between the observed data $d_{o b s}$ and the synthetic data $d_{\text {syn }}=A m$ (as in equation (8.3.1)) are being considered. The data and the normal equation relative residual norms can be computed by

$$
r_{\text {data }}=\frac{\left\|A m^{(k)}-d_{o b s}\right\|_{2}}{\left\|d_{o b s}\right\|_{2}}, \quad r_{\text {normal }}=\frac{\left\|A^{T}\left(A m^{(k)}-d_{o b s}\right)\right\|_{2}}{\left\|A^{T} d_{o b s}\right\|_{2}} .
$$

In Figure 2(c) and (d), we can observe that the relative residual error norms are decreasing and stabilising. This decreasing in residual norms show that our algorithm is converging towards the optimal MAP model concerning the synthetic data $d_{\text {syn }}=A m+e$ fits the observed data $d_{o b s}$.

Our second task is to demonstrate the concept of the optimal low-rank approximation of a posterior covariance and resolution matrices, as discussed in section 8.7 and 8.8. In a linear inverse problem setting, the posterior covariance matrix or the Hessian is not depending on the model parameter, and this simplifies the computation for quantifying the uncertainties. For this task, we compute the posterior covariance based on equation (8.6.5), then truncate its singular spectrum based on the optimal criterion given by 

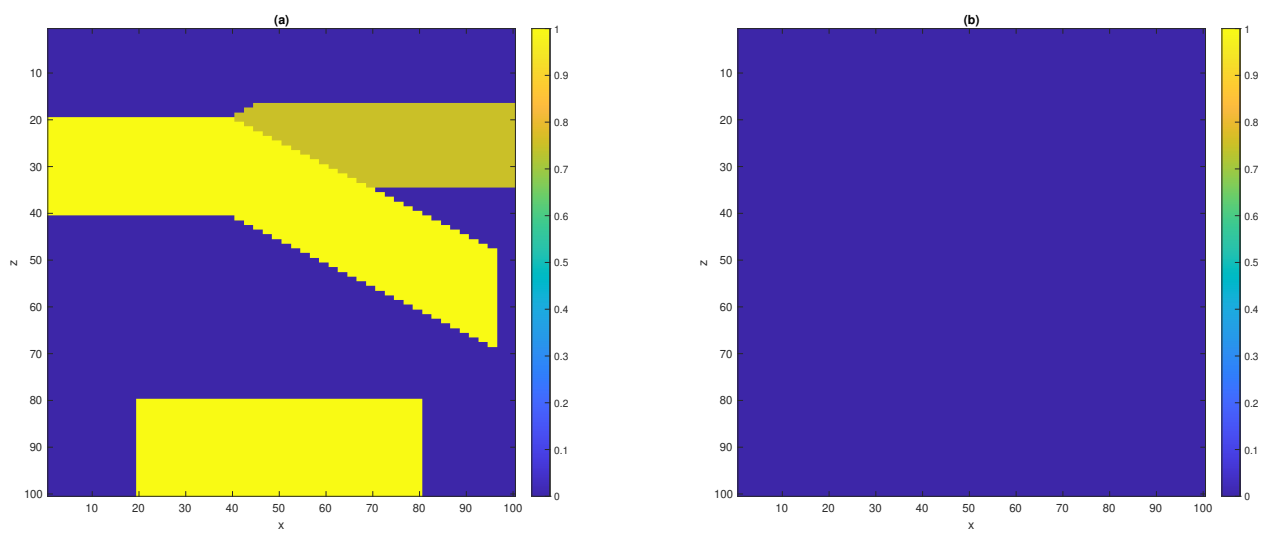

Figure 8.1: (a) True slowness model. (b) Initial slowness model

equation (8.8.1). For this specific example, the optimal truncation point is at singular value equals unity. In Figure 3, we plot the singular spectrum for the prior covariance, the Hessian, the posterior covariance, and the normalised data misfit Hessian $\left(\tilde{A}^{T} \tilde{A}\right.$ as in equation (8.5.4)), respectively. We could observe the fast decaying spectrum from all the singular values plots; hence this informs us that the data are informative only on a low-dimensional subspace.

Figure 4 (top row) shows the convergence of the approximate posterior variance for different value of singular value truncation. The first column in Figure 4 represents the prior variance and its approximation of the mean model without information from the observed data. For this formally 10,000-dimensional problem, a good approximation of the posterior variance is achieved with optimal truncation at the 437-th singular values of the the normalised data misfit Hessian $\left(\tilde{A}^{T} \tilde{A}\right)$; hence the data are informative only on a low-dimensional subspace. The quality of the covariance matrix approximation is also reflected in the structure of mean model approximation from the approximate posterior covariance (bottom row). All three of these approximated mean model are significantly close to the MAP model computed by minimising the negative logarithm of the posterior distribution. Already with truncation at singular value approximately unity (at the 437th singular values), the features of the approximate posterior variance and mean model match those of the MAP posterior variance and mean model. In applications, agreement in this variance and mean model are important. Based on this numerical example, we observed that the optimal truncation point for obtaining the low-rank approximation of a posteriori covariance can be determined by the filter factor (equation (8.8.1)) introduced in section 8.8 . 

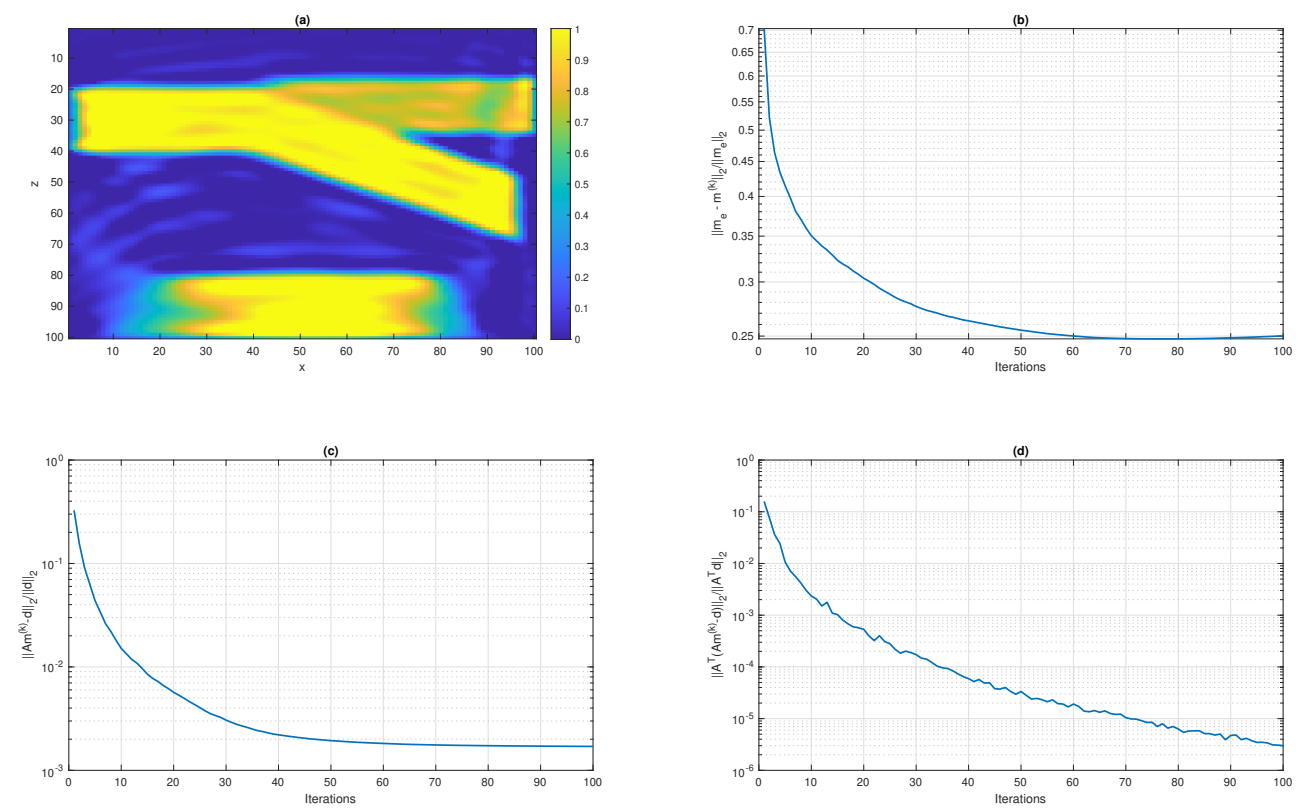

Figure 8.2: (a) The MAP model after 100 iterations of conjugate gradient. (b) The relative model error norm at each iteration. (c) The relative residual norm at each iteration. (d) The normal equation relative residual norm at each iteration.

\subsection{Conclusions}

This chapter has presented and demonstrated the sound connection between the Bayesian approach and the Tikhonov regularisation within Gaussian framework for linear inverse problems. We discussed the Tikhonov regularisation technique from a functional point of view. By connection between Bayesian approach and Tikhonov regularisation technique, we provided an uncertainty analysis framework to answer the following two fundamental questions: (1) How well is the estimate determined by the a posteriori PDF, i.e. by the combination of observed data and a priori information? (2) What are the respective contributions of observed data and a priori information? We also discussed the concept of the resolution matrix and introduced the optimal low-rank approximation of a posteriori covariance matrix for the Bayesian solution of linear inverse problems. We demonstrated it through the seismic travel-time tomography example, with Gaussian prior and noise distributions defined on finite-dimensional spaces. In a typical large-scale inverse problem, e.g. seismic inversions, observations may be informative relative to the prior only on a low dimensional subspace of parameter space. An important task that needs to be done in the future is to generalise this framework for inverse problems with nonlinear forward modelling, i.e., nonlinear inverse problems. 

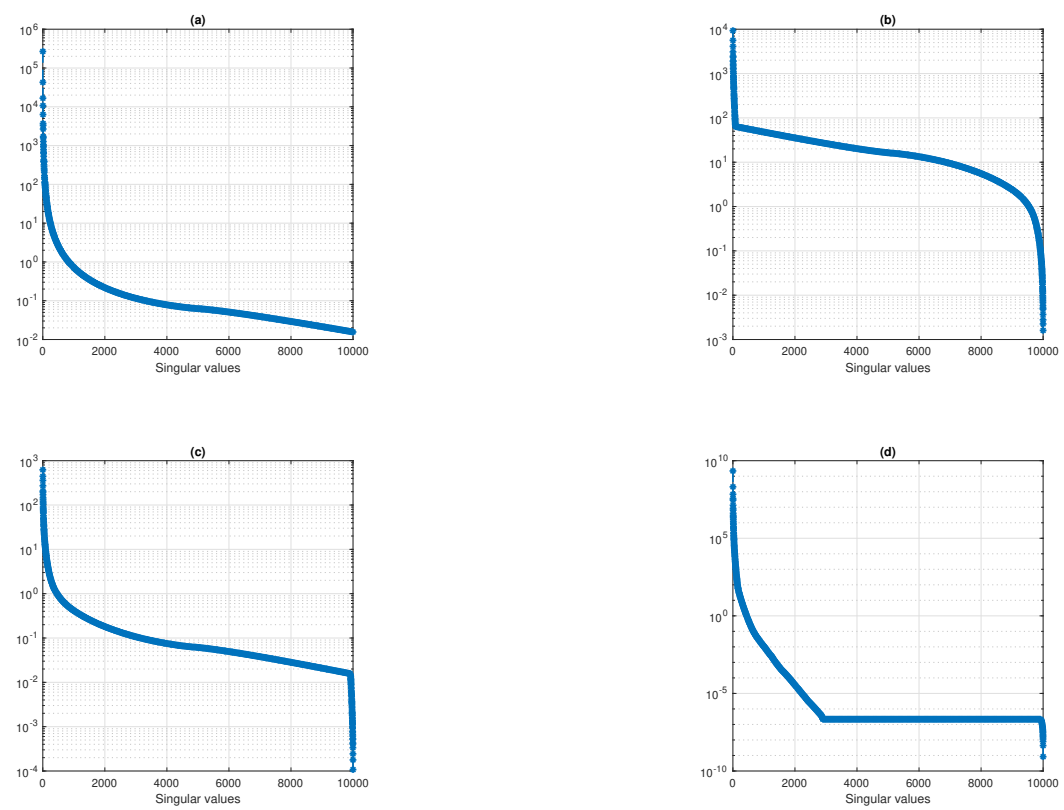

Figure 8.3: The singular values for (a) prior covariance, (b) Hessian, (c) posterior covariance, and (d) normalised data misfit Hessian.
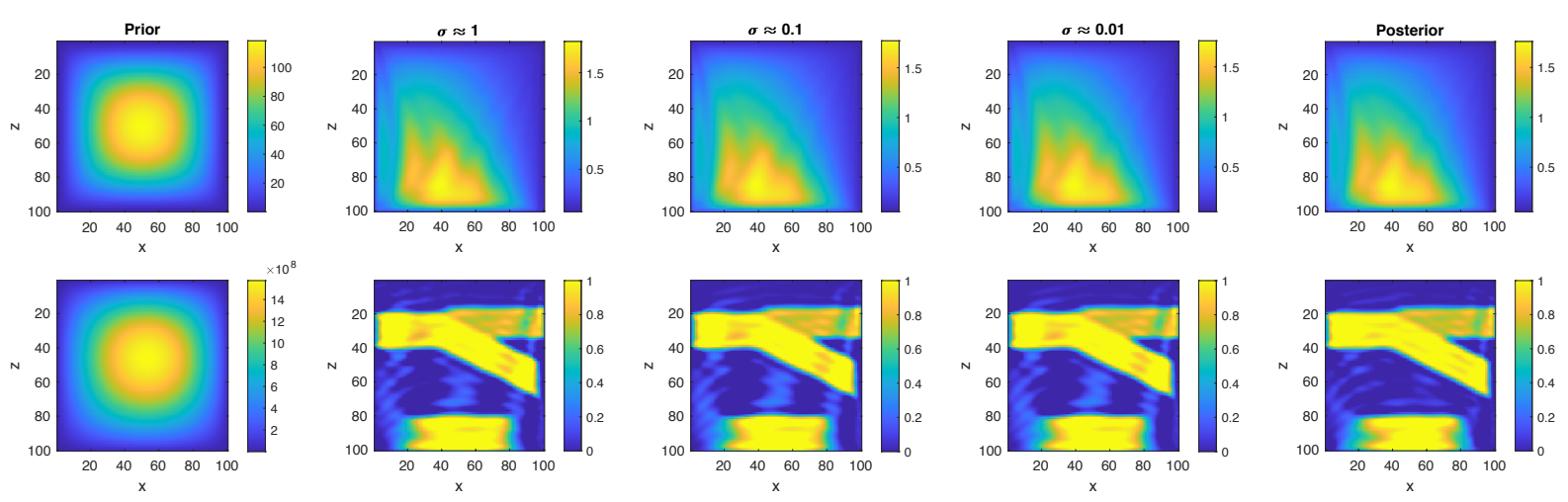

Figure 8.4: First column: Prior variance field, and its approximation of the mean model without information from the observed data. (bottom). Second through last columns (left to right): Variance field of the approximate posterior as the number of singular spectrum truncation increases (top); mean model approximation from the corresponding approximate posterior covariance (bottom) assuming exact knowledge of the posterior mean. 


\section{Bibliography}

[1] Tikhonov A. N. and Arsenin V.Ya. Methods for the Solution of Ill-posed Problems. Nauka, Moscow, 1974.

[2] Tikhonov A. N. and Arsenin V.Ya. Solutions of Ill-posed Problems. John Wiley \& Sons, New York-Toronto-London, 1977.

[3] Ivanov V. K., Vasin V.V., and Tanana V. P. Theory of Linear Ill-posed Problems and its Applications. Inverse and Ill-posed Problems Series, VSP, Utrecht, 2002.

[4] Lavrentev M. M., Romanov V. G., and Shishatskii S. P. Ill-posed Problems of Mathematical Physics and Analysis. Amer. Math. Soc., Providence, RI., 1986.

[5] Denisov A. M. Introduction to the Theory of Inverse and Ill-posed Problems. Moskov. Gos. Univ., Moscow., 1994.

[6] Kabanikhin S.I. Inverse and Ill-posed Problems. Theory and Applications. de Gruyter, 2011.

[7] Tikhonov A. N., Leonov A.S., and Yagola A.G. Nonlinear Ill-posed Problems. Nauka, Moscow, 1995.

[8] J. Martin, L. Wilcox, C. Burstedde, and O. Ghattas. A stochastic newton mcmc method for large-scale statistical inverse problems with application to seismic inversion. SIAM Journal on Scientific Computing, 34(3):A1460-A1487, 2012.

[9] T. Bui-Thanh, O. Ghattas, J. Martin, and G. Stadler. A computational framework for infinite-dimensional bayesian inverse problems part i: The linearized case, with application to global seismic inversion. SIAM Journal on Scientific Computing, 35(6):A2494-A2523, 2013.

[10] Noemi Petra, James Martin, Georg Stadler, and Omar Ghattas. A Computational Framework for Infinite-Dimensional Bayesian Inverse Problems, Part II: Stochastic Newton MCMC with Application to Ice Sheet Flow Inverse Problems. SIAM Journal on Scientific Computing, 2014.

[11] L. Métivier, R. Brossier, S. Operto, and J. Virieux. Full Waveform Inversion and the Truncated Newton Method. SIAM Review, 2017.

[12] Zhilong Fang, Curt Da Silva, Rachel Kuske, and Felix J. Herrmann. Uncertainty quantification for inverse problems with weak partial-differential-equation constraints. GEOPHYSICS, 2018. 
[13] Andreas Fichtner and Jeannot Trampert. Resolution analysis in full waveform inversion. Geophysical Journal International, 2011.

[14] Jeannot Trampert, Andreas Fichtner, and Jeroen Ritsema. Resolution tests revisited: The power of random numbers. Geophysical Journal International, 2013.

[15] Andreas Fichtner and Tristan Van Leeuwen. Resolution analysis by random probing. Journal of Geophysical Research: Solid Earth, 2015.

[16] G. Backus and F. Gilbert. The resolving power of gross earth data. Geophysical Journal of the Royal Astronomical Society, 16(2):169-205, 1968.

[17] G. Backus and F. Gilbert. Uniqueness in the inversion of inaccurate gross earth data. Philosophical Transactions of the Royal Society of London. Series A, Mathematical and Physical Sciences, 266(1173):123-192, 1970.

[18] A. Tarantola and B. Valette. Inverse problems = quest for information. Journal of geophysics, 50(1):159-170, 1982.

[19] A. Tarantola and B. Valette. Generalized nonlinear inverse problems solved using the least squares criterion. Reviews of Geophysics, 20(2):219-232, 1982.

[20] A. Tarantola. Inverse Problem Theory and Methods for Model Parameter Estimation. Society for Industrial and Applied Mathematics, 2005.

[21] A. J. W. Duijndam. Bayesian estimation in seismic inversion. part i: Principles. Geophysical Prospecting, 36(8):878-898, 1988.

[22] A. J. W. Duijndam. Bayesian estimation in seismic inversion. part ii: Uncertainty analysis. Geophysical Prospecting, 36(8):899-918, 1988.

[23] H. Flath, L. Wilcox, V. Akçelik, J. Hill, B. van Bloemen Waanders, and O. Ghattas. Fast algorithms for bayesian uncertainty quantification in large-scale linear inverse problems based on low-rank partial hessian approximations. SIAM Journal on Scientific Computing, 33(1):407-432, 2011.

[24] T Cui, J Martin, Y M Marzouk, A Solonen, and A Spantini. Likelihood-informed dimension reduction for nonlinear inverse problems. Inverse Problems, 30(11):114015, oct 2014 .

[25] A. Spantini, A. Solonen, T. Cui, J. Martin, L. Tenorio, and Y. Marzouk. Optimal low-rank approximations of bayesian linear inverse problems. SIAM Journal on Scientific Computing, 37(6):A2451-A2487, 2015.

[26] Klaus Mosegaard and Albert Tarantola. Monte carlo sampling of solutions to inverse problems. Journal of Geophysical Research: Solid Earth, 100(B7):12431-12447, 1995.

[27] Steve Brooks, Andrew Gelman, Galin Jones, and Xiao-Li Meng. Handbook of Markov Chain Monte Carlo. CRC press, 2011.

[28] David J. C. MacKay. Information Theory, Inference Learning Algorithms. Cambridge University Press, USA, 2002. 
[29] Daniela Calvetti and Erkki Somersalo. Introduction to Bayesian Scientific Computing: Ten Lectures on Subjective Computing (Surveys and Tutorials in the Applied Mathematical Sciences). Springer-Verlag, Berlin, Heidelberg, 2007.

[30] Jari Kaipio and Erkki Somersalo. Statistical inverse problems: Discretization, model reduction and inverse crimes. J. Comput. Appl. Math., 198(2):493-504, January 2007.

[31] P. Hansen. Rank-Deficient and Discrete Ill-Posed Problems. Society for Industrial and Applied Mathematics, 1998.

[32] Heinz W. Engl, Martin Hanke, and Andreas Neubauer. Regularization of inverse problems. Kluwer Academic Publishers, 2000.

[33] P. Hansen. Discrete Inverse Problems. Society for Industrial and Applied Mathematics, 2010.

[34] S. I. Kabanikhin. Inverse and ill-posed problems: theory and applications. De Gruyter, 2012.

[35] Silvia Gazzola, Per Christian Hansen, and James G. Nagy. Ir tools: a matlab package of iterative regularization methods and large-scale test problems. Numerical Algorithms, 81(3):773-811, 2019. 